Caligrama, Belo Horizonte, v. 21, n. 2, p. 95-111, 2016

\title{
Langue, culture et didactique du FLE
}

\section{Language, culture and FFL didactics}

Christianne Benatti Rochebois

Universidade Federal do Sul da Bahia (UFSB), Porto Seguro / Brasil

chrisrochebois@hotmail.fr

Résumé: Ce texte présente des réflexions basées sur l'étude des principales méthodologies de l'enseignement de langue étrangère - de la méthode traditionnelle à l'époque actionnelle - et comment y sont évoqués les diverses manières de concevoir la langue et la culture au fil du temps. Considérant que la pluralité des contextes d'interaction explique le caractère pluriel et instable de toute culture et que la langue est le produit de l'interaction entre ceux qui la parlent, la culture n'existe que par le jeu interactif des individus. Ainsi, langue et culture s'actualisent, construisent des significations et présentent leur dynamique dans la diversité des espaces, des relations et des interactions sociales, lieu où la langue, la culture et les représentations interagissent, communiquent et produisent du sens.

Mots-clé: représentations culturelles; interactions sociales; méthodologies d'enseignement-apprentissage.

Abstract: This text presents thoughts based on the study of the main methodologies for the teaching of French as a foreign language - from the traditional method to the era of the "actionelle" approach - and on how, as time goes by, different ways of conceiving language and culture are evoked in these methodologies. Considering that the plurality of interactional contexts explains the multifarious and unstable character of a culture, and that language is the product of the interaction among those 
that speak it, culture does not exist but by the interactional game of the individuals. Therefore, language and culture feed each other, build their meanings and show a dynamics at the diversity of spaces, relations and social interactions, where language, culture, and representations interact, communicate and make sense.

Keywords: cultural representations; social interactions; teachinglearning methodologies.

Recebido em: 30 de agosto de 2016.

Aprovado em: 6 de outubro de 2016.

\section{Introduction}

L'intérêt que nous accordons à l'étude de la construction des représentations culturelles dans le processus d'enseignement/ apprentissage d'une langue étrangère nous a conduit à chercher à repérer l'évolution des conceptions de la notion de culture, de l'origine latine jusqu'aux définitions scientifiques contemporaines. Cet examen nous a conduite à montrer comment la langue en est l'un des éléments constitutifs. Nous supposons que dans la didactique du FLE (Français Langue Étrangère), la construction des représentations culturelles est liée à trois facteurs:

- la conception de la culture des manuels de FLE révélée par le choix des éléments culturels abordés;

- la conception de la culture française des professeurs non natifs de FLE (ainsi que les institutions où ils travaillent) et leurs représentations de la culture enseignée.

- l'approche méthodologique des professeurs dans le traitement des documents destinés à la présentation de l'autre culture.

Notre travail de recherche est centré sur la diversité culturelle telle qu'elle apparaît dans les documents destinés à l'étude d'une nouvelle culture, dans notre cas la culture francophone dans les manuels de FLE. Le rôle du professeur nous paraît fondamental dans la mesure où le processus de découverte culturelle dépend de la conception qu'il a de la culture francophone et de ses représentations de la communauté travaillée. 
La langue comme élément constitutif de la culture et partie intégrante du processus d'accès à une culture étrangère, joue son rôle dans l'élaboration du discours inducteur de représentations culturelles. Son analyse peut aider l'enseignant à assumer auprès des élèves une fonction de médiateur entre deux cultures.

Pour la mener à bien, son intervention devrait viser la prise de conscience par l'apprenant que le discours est traversé de représentations stéréotypées. Ces représentations tendent à survaloriser ou à dénigrer certains aspects d'une communauté culturelle déterminée, parfois au détriment de la culture de l'apprenant. Il nous apparaît important de traiter les différences culturelles en tant que réalités historiques et socio culturelles distinctes et contextualisées dans le temps et dans l'espace. Dans cette perspective, l'enseignant, conscient de son rôle de médiateur et de formateur, devrait être muni d'éléments concrets construits sur une analyse attentive et critique du discours et sur une variété de documents issus de différentes sources, sans se baser seulement sur ses expériences personnelles de contact avec l'autre culture.

La description de la réalité culturelle étrangère à partir d'éléments empiriques est dénoncée par Zarate (1986, p. 29) comme une erreur méthodologique transformant une expérience individuelle en vérité générale:

[...] Faute d'être reconnue et analysée (la perception des faits culturels de la part des enseignants) comme un point de vue spécifique, l'opinion se transforme en jugement absolu: 'les Français sont...'. Ces jugements à l'emportepièce, si fréquents dans la conversation quotidienne, sont autant de lampes rouges qui signalent l'erreur de méthode. L'abus consiste à transformer une expérience individuelle en vérité générale $[. .$.

Pour parvenir à une description objective de la culture étrangère, l'enseignant non-natif devrait considérer, dans son analyse descriptive, plusieurs éléments qui, selon Zarate (1986, p. 29), sont d'une extrême importance pour que les expériences individuelles ne deviennent pas des vérités générales:

Tout discours contient le point de vue de celui qui l'énonce [...] Cela s'applique dans toute sa rigueur à l'enseignant non français qui, après un contact de courte, moyenne ou 
longue durée, rend compte en classe de la réalité française (ou francophone). Dans la relation qu'il en fera à ses élèves, en quoi sa description reste-t-elle dépendante de son expérience singulière et partiale? Un séjour de boursier ou de touriste, une expérience d'homme ou de femme, des origines provinciales ou parisiennes, un contact en milieu rural ou urbain, des amis étudiants ou aux prises avec la vie professionnelle sont pour l'enseignant de français langue étrangère autant de variables qui peuvent intervenir dans sa perception des faits culturels [...]

Cette analyse montre bien que l'intervention de l'enseignant peut être, dans certains cas, considérée davantage comme celle d'un témoin particulier d'une expérience spécifique vécue, que comme celle d'un médiateur culturel entre sa réalité et celle de ses élèves avec les étrangers, l'enseignant étant lui-même porteur de ses propres représentations.

Une culture étrangère appréhendée de diverses façons peut susciter chez l'apprenant la prise de conscience de la relativité des points des vue et des expériences. Il y a là une voie qui conduit à comprendre l'autre et aussi à se redécouvrir soi-même, puisque le regard extérieur sur une réalité culturelle étrangère favorise chez l'observateur sensibilisé à la diversité culturelle, un retour sur lui-même et sur ses propres croyances. Une objectivation de sa propre culture, de ses références et pratiques culturelles est ainsi rendue possible. Ce retour sur soi-même peut enseigner à respecter ces différences autant dans le macro que dans le micro univers de nos relations.

La déclaration de Trotot (1998) dans la présentation du livre de Jean de Léry - Histoire d'un voyage fait en la terre de Brésil (1578), illustre ce mouvement de façon éclairante: 'Or, s'interroger sur l'autre c'est s'interroger sur ce qui me sépare de l'autre, mais aussi sur ce qui m'en rapproche. Ce qui revient à ajouter à la question 'qui est cet autre homme que je rencontre?' 'qui suis-je, moi qui le rencontre?'” (TROTOT, 1998, p. 10).

L'effort pour apprendre une culture étrangère n'est pas un thème récent dans la didactique des langues étrangères. Depuis le XIX ${ }^{\text {ème }}$ siècle, on cherche, à partir de l'enseignement des langues, à aborder et pénétrer la culture à partir de la littérature et des arts d'une façon générale, pour enrichir l'esprit. Cette conception de l'enseignement de la culture a eu une longue vie et a perduré jusqu'à la fin du $\mathrm{XX}^{\text {ème }}$ siècle 
avec peu de changements. Cette conception privilégie les connaissances informatives et factuelles qui contribuent très peu à la construction d'une compétence culturelle. La question de cette compétence culturelle nous semble d'autant plus cruciale qu'elle est liée à celle de la formation des enseignants. L'étude des principales méthodologies de l'enseignement de langue étrangère, va nous permettre d'évoquer les diverses manières de concevoir la langue et la culture telles qu'elles sont apparues au fil du temps.

\section{Culture / Littérature}

Dans la période où la méthode traditionnelle ou classique a prédominé, la langue était définie comme "un ensemble de règles et d'exceptions observables dans des phrases ou des textes" (GERMAIN, 2010, p. 13), et son enseignement basé sur l'étude de textes littéraires. La grammaire et la traduction étaient privilégiées et l'accent mis sur la lecture et la compréhension de textes et, pour ce qui concerne l'écrit, sur la rédaction. L'objectif principal était de "rendre l'apprenant capable de lire les ouvrages littéraires écrits dans la langue cible [...] et de développer ses capacités intellectuelles" (GERMAIN, 2010, p. 12). On considérait ainsi que l'accès à la culture étrangère passait par la connaissance de la littérature et des arts. La démarche prenait appui sur une conception de la culture synonyme d'enrichissement de l'esprit grâce à l'acquisition de connaissances tirées de la production littéraire et artistique d'un peuple.

D'après Porcher (2004), on observe aujourd'hui une tendance à réduire ce type d'enseignement sous prétexte que les apprenants ne s'y intéressent pas vraiment. Il soutient que le système éducatif doit se préoccuper de cet aspect de la culture qui fait partie de la culture nationale en tant que patrimoine culturel:

... l'institution scolaire doit impérativement transmettre cette tradition parce qu'elle reflète une culture nationale tout entière. Que cet enseignement soit modernisé, certes, mais il faut qu'il tienne la place (également par les médias) qu'il représente effectivement pour la culture étrangère des apprenants (PORCHER, 2004, p. 54).

L'hégémonie de la langue française en Europe pendant les XVIII ${ }^{\text {ème }}$ et XIX ${ }^{\text {ème }}$ siècles a conforté cette conviction de l'époque qu'apprendre 
le français et la culture française (comprise comme littérature et art), permettait de gravir l'échelle sociale et de se différencier des autres. Parlé par tous les souverains européens, le français était la langue de l'élite, symbole d'une civilisation supérieure et doté du pouvoir d'éclairer les idées et la pensée. Cette conception de la langue française comme langue d'une élite s'est perpétuée dans le temps jusqu'à la première moitié du

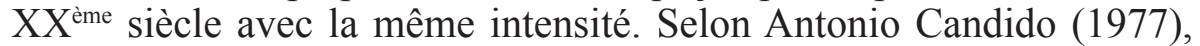
la France a toujours joué un rôle de médiation culturelle aux yeux des Brésiliens, «grâce à elle nous avons pu voir le monde, nous avons acquis le sens de l'Histoire, nous avons lu les classiques de tous les pays, inclus les Grecs et les Romains» (CANDIDO, 1977, p. 12, notre traduction). ${ }^{1}$ Toujours selon Candido (1977, p. 10, notre traduction), elle nous (à nous, les Brésiliens) a offert «la possibilité de nous mettre en contact avec d'autres cultures». ${ }^{2}$

À la fin du XIX ${ }^{\text {ème }}$ siècle et début du $X^{\text {ème }}$ siècle, on a vu renaître la méthode directe dont l'objectif était d'enseigner la langue pour communiquer. Sa caractéristique principale, était de donner la priorité à la langue orale quotidienne, les cours étant donnés dans la languecible. On pouvait penser que, de la sorte, les enseignants amèneraient les apprenants à penser dans la langue étrangère par association d'idées, en se servant d'images, d'objets, de gestes et de la mimique. La langue était conçue comme un instrument de communication orale, et la culture n'était plus synonyme de littérature et d'arts. On va commencer alors à se rendre compte que l'enseignement de la culture (même d'une façon indirecte), passe par "l'étude des valeurs culturelles (par exemple, le mode de vie du quotidien des natifs de L2) aussi bien que la géographie et l'histoire" (GERMAIN, 2010, p. 128). Malgré leur caractère artificiel, les dialogues sont insérés dans un contexte ou une situation (la poste, la banque, le restaurant...). Ce sont donc les aspects culturels les plus quotidiens qui apparaissent de manière prédominante et appellés dans son ensemble comme aspects de la civilisation française.

Porcher, dans son article "L'enseignement de la civilisation", nous dresse 1'historique et la discussion sur les termes "culture" et "civilisation" dans la didactique des langues, conférant ce dernier

\footnotetext{
1 “[...] graças a ela que pudemos ver o mundo, que adquirimos o senso da História, que lemos os clássicos de todos os países, inclusive gregos e romanos."

2 "[...] a possibilidade de nos pormos em contato com outras culturas".
} 
“comme quelque chose à voir avec les représentations que l'on se fait de l'étranger" (PORCHER, 1994, p. 5). Dans les manuels de FLE qui utilisent le mot "civilisation", on présente souvent des paysages, des artistes, de la mode, enfin, l'étudiant n'est jamais confronté à des problèmes, aux difficultés de la vie des immigrants par exemple, à la violence dans la banlieue de Paris. Les éléments y sont abordés de façon superficielle, avec un message idéaliste de la France.

Les avancés technologiques et scientifiques et le développement des sciences du langage dans la première moitié du $X{ }^{\text {ème }}$ siècle ont beaucoup contribué à l'évolution de l'enseignement des langues étrangères.

À partir des découvertes de la psychologie béhavioriste de la linguistique structurale, la méthode Audio-Orale apparaît au milieu des années 1950. D’origine américaine, elle concevait la langue comme un ensemble d'habitudes et son apprentissage comme la création d'automatismes linguistiques. Son objectif était d'amener l'apprenant à communiquer dans la langue étrangère suivant l'ordre d'acquisition de langue maternelle, c'est-à-dire, la compréhension orale, l'expression orale, la compréhension écrite et l'expression écrite. L'interdiction de l'utilisation de la LM dans la salle de classe n'empêchait pas l'analyse comparée de deux langues (LM et LE). Les éléments culturels traités par les manuels de la langue présentent la façon de vivre des membres de la société cible.

Germain (1993, p. 111) définit la conception de la culture en vigueur comme "l'ensemble de croyances et des modes de comportement d'un groupe social, tels que réfléchis dans les arts et les métiers artisanaux, dans les contes et les mythes, dans le travail et dans le jeu, dans la religion et dans la vie quotidienne [...]". Dans cette définition, on observe un élargissement dans la conception de la culture avec la prise en compte de la culture quotidienne. Ce surcroît ou ce complément apportés par Germain à la conception de la culture montrent une évolution de la notion appliquée à l'enseignement des langues étrangères; c'est une ouverture et un premier pas vers le sens sociologique et non simplement littéraire et artistique du terme. Ainsi l'auteur déclare-t-il:

Il ressort clairement de notre analyse [...] que le professeur doit rattacher la langue à la culture [...]. Telle est la conclusion de Robert Politzer, qui écrit [...]: 'En tant que professeurs de langue nous devons nous intéresser à l'étude de la culture (dans le sens sociologique du terme), 
non pas parce que nous voulons enseigner nécessairement la culture de l'autre pays mais parce que nous devons l'enseigner. Si nous enseignons une langue sans enseigner en même temps la culture dans laquelle elle s'insère, nous enseignons des signes dépourvus de sens ou des signes auxquels l'étudiant attribue une signification erronée [...] Germain (1993, p. 149).

Même conçue de manière statique, l'association de l'enseignement de la langue à l'enseignement de la culture représente une avancée méthodologique dans la didactique de langue étrangère.

Avec le relatif échec de la méthode Audio-Orale dû, notamment, à l'incapacité des apprenants de s'exprimer dans la langue étrangère à la fin d'un nombre raisonnable d'heures de cours, la méthode suivante, SGAV (Structuro-Global Audio Visuel) ${ }^{3}$ apparaît. C'est une méthode à dominante orale, qui s'appuie sur la compréhension de dialogues centrés sur des sujets de la vie quotidienne, et qui présente les éléments culturels de façon explicite ou implicite, des éléments qui peuvent faire référence à la manière de vivre des Français et aussi à leur littérature.

La publication du manuel C'est le printemps (J. Montredon et al., Clé International, Paris, 1976), sorte d'intermédiaire dans le passage du SGAV pour l'approche communicative, a suscité plusieurs critiques à propos des innovations d'ordre culturel présentées, par exemple, l'introduction de sujets polémiques comme le racisme. Ce manuel a connu une existence brève; s'il ne véhiculait pas une image idyllique de la France, il a cependant constitué une étape dans l'évolution des méthodologies en proposant un traitement critique et plus réaliste de la culture française:

Aussi (C'est le printemps) n'est-il pas histoire d'une famille petite bourgeoise sans problèmes ou une idylle entre jeunes gens timides, mais une suite de situations mettant en scène des personnages divers, des gens de tous âges, des Français et même des étrangers vivant

\footnotetext{
${ }^{3}$ Sous la responsabilité de Peter Guberina, de l'Université du Zagreb et de Paul Rivenc, de l'École Normale Supérieure de Saint-Cloud, naît VIF (Voix et Images de France), manuel didactique destiné à l'enseignement du français pour les enfants de 8 à 11 ans, basé sur la notion de «structuro-global» (le mot en situation) et sur la théorie verbotonale de Guberina (la théorie de la perception auditive et visuelle).
} 
en France. Par eux, à travers eux, une certaine réalité française apparaît. [...] 'C'est le printemps' est également né d'une critique d'ordre linguistique. [...] La progression linguistique des six premières unités vise à l'autonomie d'apprentissage de l'étudiant, ainsi que d'acquisitions de moyens linguistiques directement utilisables en situation de communication. (MONTREDON et al., 1976, p. 5, livre du professeur apud DE CARLO; LANCIEN, 1998, p. 32)

À la fin des années 1960 et au début des années 1970, la didactique des langues étrangères doit faire face à une nouvelle réalité sociale, marquée par la grande ouverture des marchés, par le développement des moyens de transport, ainsi que par l'extension des progrès technologiques dans le monde développé. Ces facteurs de mobilité et de communication entre les cultures, ont ouvert l'enseignement des langues étrangères sur les besoins linguistiques professionnels et sur la formation académique. L'accès à la littérature spécifique et aux publications scientifiques est en effet de plus en plus nécessaire, et impose une conception rénovée de l'enseignement des langues étrangères, tant sur le plan linguistique que sur le plan pédagogique. Un effort d'adéquation aux besoins s'est avéré nécessaire pour répondre à cette nouvelle demande du marché: Il ne s'agit plus d'enseigner le français général, mais le français centré sur les spécificités locales et de public, le français transmetteur d'informations; c'est la rédemption du français écrit, la naissance du Français Instrumental. Le grand changement a été le centrage de l'enseignement des langues étrangères sur les besoins de l'apprenant adulte, en prenant en considération des facteurs externes jusqu'alors négligés, comme sa disponibilité en temps, ses objectifs, ses attentes et ses caractéristiques personnelles:

Cette pratique correspond aux choix précis d'objectifs, de contenus et de méthodes, motivés par la situation des pays en question et par les nécessités présentes et futures de leurs étudiants $[\ldots]$ Le français instrumental [...] vise l'enseignement massif aux étudiants qui ont commencé une discipline universitaire [...] Il s'agit de fournir un outil de travail à l'étudiant de n'importe quel domaine [...] avec l'objectif de le rendre capable de comprendre le contenu d'un texte, d'extraire l'information et éventuellement, de la transmettre à d'autres personnes dans sa langue maternelle 
[...] Il est supposé que le niveau élémentaire existe déjà (de langue des étudiants), soit par un cours de français traditionnel, ou d'un cours VIF ou un autre quelconque (ALVAREZ, 1976, p. 7).

L'enseignement de la langue et, par conséquent, de la culture, s'est ainsi trouvé associé, à la divulgation des progrès technologiques et scientifiques du pays de la L2.

Simultanément, les progrès dans les divers domaines des sciences du langage ont contribué à l'évolution des conceptions de la langue et de la culture dans la didactique de langues étrangères. On peut notamment souligner, la prise en compte des aspects sociaux du langage revendiquée par Hymes, les réflexions sur le langage comme instrument de communication, de Austin et Searle, l'intérêt de plusieurs auteurs pour les aspects sémantiques du langage, avec le diagnostic de besoins linguistiques nouveaux et différents d'un locuteur à l'autre dans le contexte étendu des relations européennes. Ces contributions sont à la base de l'approche communicative qui commence à naître, comme l'expose Germain (1993, p. 202):

C'est la convergence de ces quelques courants de recherche ainsi que l'avènement de différents besoins linguistiques dans le cadre d'une Europe élargie (marché commun, Conseil de l'Europe, etc) qui a en définitive donné naissance à l'approche communicative [...] L'événement marquant qui allait donner l'occasion à quelques chercheurs de fondre en un tout l'ensemble de courants théoriques convergents [...] est d'ordre politique [...] et en 1972, le Conseil de l'Europe réunissait un groupe d'experts chargés de mettre sur pied des cours de langues pour adultes.

Apparaît ainsi, la notion de compétence de communication qui intègre, en plus de la compétence grammaticale, la compétence sociolinguistique et la compétence stratégique (des stratégies verbales et non-verbales). La langue est comprise tant comme un instrument de communication que comme élément d'interaction sociale. L'objectif principal est de conduire l'apprenant à communiquer de manière efficace dans la L2, en étant capable d'adapter les registres de langue à la situation de communication en fonction de son intention. Dans cette perspective, la connaissance des règles grammaticales ne peut suffire pour acquérir cette 
compétence de communication. Ce qui est visé, c'est le développement de la capacité à récréer la langue, à découvrir de nouvelles règles de formation d'énoncés, l'élève participant ainsi à son propre apprentissage, selon la psychologie cognitive.

Le but général est d'en arriver à ce que les apprenants communiquent de façon efficace [...] les tenants de l'approche communicative considèrent qu'une communication efficace implique une adaptation des formes linguistiques à la situation de communication (statut de l'interlocuteur, âge, rang social, lieu physique, etc.) et à l'intention de communication (ou fonction langagière: demander d'identifier un objet, demander une permission, donner des ordres, etc.) (GERMAIN, 1993, p. 203).

La culture, pour ce qui concerne cet aspect, est conçue de manière plus dynamique mettant en valeur les faits de la vie quotidienne, le comportement et les éléments non-verbaux présents dans toute communication linguistique: "Par culture, l'approche communicative fait surtout référence à la vie quotidienne, en tenant compte du comportement non-verbal qui accompagne toute communication linguistique" (GERMAIN, 1993, p. 201).

L'approche communicative n'offre pas de recettes et de formules à suivre comme garanties pour réussir, mais elle se base sur des principes fondamentaux qui doivent être pris en considération par le professeur devant son groupe d'élèves. Avec l'introduction de la notion d'intention de communication associée à une situation, l'apprenant est exposé plus tôt à un ensemble de possibilités variées pour une seule prise de parole ou fonction langagière. La sensibilisation à ces éléments modifie la conception de la langue et l'insère dans un mouvement plus dynamique que dans la méthode SGAV. L'éventail des possibilités présenté permet que l'apprenant commence, par lui-même, à récréer la langue et à ajuster ces variantes à des contextes spécifiques.

Dans une perspective assez similaire, l'époque actionnelle commence à partir de la moitié des années 1990, en accompagnement de l'évolution politique, économique et sociale mondiale. Prenant prioritairement en compte les nécessités d'une Europe unifiée, ce courant, le plus récent dans le champ de la didactique des langues étrangères, vise la préparation des Européens à une convivialité sociale et à l'intégration professionnelle. L'ambition de la perspective actionnelle est la formation 
d'apprenants non seulement capables de parler et de comprendre une L2, mais aussi d'agir et réagir, d'utiliser cette langue dans une autre société et de prendre en considération la diversité culturelle. La conception de langue qui la sous-tend est celle d'un "outil d'action commune et non plus seulement de communication réciproque" (PUREN, 2001, p. 9-10).

Le résultat majeur de toutes ces questions, considérations et constatations pour l'enseignement de langues est la publication du Cadre Européen Commun de Référence pour l'apprentissage et l'enseignement des Langues (élaboré par le Comité de l'Éducation du Conseil de l'Europe, en 1996) qui considère avant tout "l'usager et l'apprenant d'une langue comme des acteurs sociaux ayant à accomplir des tâches (qui ne sont pas seulement langagières) dans des circonstances et un environnement donné, à l'intérieur d'un domaine d'action particulier" (CONSEIL DE L'EUROPE, 2001, chap. 2, p. 15).

Ce document de référence, important pour l'enseignement/ apprentissage des langues vivantes, conçoit l'usage de la langue et son apprentissage comme

des actions accomplies par des gens qui, comme individus et acteurs sociaux, développent un ensemble de compétences générales et, notamment une compétence à communiquer langagièrement. Ils mettent en oeuvre les compétences dont ils disposent dans des contextes et des conditions variés et en se pliant à différentes contraintes afin de réaliser des activités langagières permettant de traiter (en réception et en production) des textes portant sur des thèmes à l'intérieur de domaines particuliers, en mobilisant les stratégies qui paraissent le mieux convenir à l'accomplissement des tâches à effectuer. Le contrôle de ces activités par les interlocuteurs conduit au renforcement ou à la modification des compétences (CONSEIL DE L'EUROPE, 2001, chap. 2, p. 15).

L'apprenant de langue devient donc selon les principes actionnels, un utilisateur de la langue, qui, en tant que tel, agira et réagira selon les contextes variés auxquels un natif est exposé. La conception de la langue n'est plus celle "d'une expression neutre de la pensée, mais du besoin et du désir de communiquer naissant d'une situation donnée dont la forme et le contenu de la communication doivent répondre à cette situation" (CONSEIL DE L'EUROPE, 2001, chap. 4, p. 41). 
L'extension du concept de culture dans la perspective actionnelle englobe tout autant la connaissance du monde de l'apprenant (connaissance qui a été acquise par l'expérience, par l'éducation, par l'information ou par d'autres moyens), son savoir socioculturel et sa conscience interculturelle. De cette façon, la connaissance de la société et de la culture de la langue étrangère travaillée, correspond à l'un des aspects des compétences visées.

La connaissance, la conscience et la compréhension des relations (ressemblances et différences distinctives) entre 'le monde d'où l'on vient' et 'le monde de la communauté cible' sont à l'origine d'une prise de conscience interculturelle [...] Elle s'enrichit également de la conscience qu'il existe un plus grand éventail de cultures que celles véhiculées par les L1 et L2 de l'apprenant [...] et englobe la conscience de la manière dont chaque communauté apparaît dans l'optique de l'autre, souvent sous la forme de stéréotypes nationaux (CONSEIL DE L'EUROPE, 2001, chap. 5, p. 83).

\section{Considérations}

A l'évidence, un apprenant, ou plus généralement un être social, n'est pas seul à construire les situations, et l'échantillon des contextes et des possibilités qu'une culture lui offre, l'amène à un univers étendu de cultures du monde et de la dimension de leurs contacts.

Selon Marie-Bernadette Le Berre (1998, p. 40), l'enseignement de la culture comprend la construction de références qui nous orientent et qui définissent nos pratiques pédagogiques. Pour cela, trois postulats constitueraient la base d'une didactique des cultures:

- Une culture se construit, c'est-à-dire, se définit comme modes de production plutôt que comme catalogue de produits;

- une compétence culturelle, comme maîtrise personnelle de ces procédés de construction, est l'objectif spécifique de l'enseignement des cultures;

- la méthodologie d'accès à cette compétence est donc fondée sur l'apprenant/producteur de sens culturel et sur une pédagogie active d'apprentissage (LE BERRE, 1998, p. 40). 
Fonder l'enseignement de la culture sur une méthodologie centrée sur l'apprenant/producteur de sens culturel signifie prendre comme point de départ sa connaissance de monde, ses expériences, perceptions et représentations afin qu'il développe sa compétence culturelle. L'objectif est non seulement d'acquérir des connaissances informatives sur la culture étrangère, mais surtout un savoir qui lui permette de comprendre le fonctionnement d'une société, et qui permette aussi de lire et de relativiser le monde au travers d'un processus de redécouverte de soi-même et de découverte de l'autre, afin qu'il puisse interagir dans ce nouveau contexte.

Nous sommes alors confrontés à l'importance des représentations dans ce processus constructeur du savoir-faire et du savoir-être en l'absence de vérités absolues ou de réalités indiscutables. Comme déclare Le Berre (1998, p. 42), "regarder les cultures des autres oblige à regarder la sienne."

L'enseignant doit lui-même avoir ces postulats présents à l'esprit; ceci implique qu'il les mette en pratique dans son propre processus de formation. Il doit avoir conscience de la diversité et de la pluralité des cultures, afin de repenser l'enseignement de la culture et fournir à ses apprenants des éléments utiles à la formation de leur compétence culturelle dans une perspective pluraliste.

Le Berre (1998) propose aux étudiants de son cours Maîtrise FLE trois lignes directrices qui fonctionnent en tant que déclencheurs de ce processus de formation et d'éveil aux autres cultures. La première est la reconnaissance de l'importance de l'anthropologie culturelle et des sciences sociales dans le processus de formation de la personne, ce qui exige de sa part, un processus de décentration et d'ouverture au monde.

La deuxième ligne directrice correspond à l'objectivation par des techniques qui aident le futur enseignant de langue à découvrir l'effet qu'il produit vraiment et non ce qu'il prétend ou imagine produire. De cette façon, objectiver sa propre action se constitue, selon Le Berre (1998, p. 43) en un

Processus méthodique, pratiquable par tout de monde, selon lequel on construit un phénomène à étudier [...]. Pour cela, on met le phénomène à distance, on le considère 'comme de l'extérieur' [...]. Les techniques d'objectivation conduisent à la construction d'un terrain commun, donc de ce qui rend possible le travail collectif et la coopération. 
Porcher (1995 apud LE BERRE, 1998, p. 43) affirme qu'objectiver sa propre action comprend également la construction d'un savoir provisoire à partir de la conscience des différences, de l'opposition et de la rupture avec des savoirs anciens.

L'importance de la mise en contexte des faits culturels permet à l'apprenant de langue étrangère et à tout individu désireux d'apprendre et de connaître d'autres cultures, de réfléchir sur le nouveau et de le comprendre dans son cadre d'origine. De même qu'il y a une nécessité à insérer un événement déterminé dans son contexte historique, de même il convient que les autres cultures soient situées dans le contexte qui les actualise en permanence afin d'en maintenir la cohérence et d'éviter le processus de folklorisation.

La mise en contexte se définit, selon Le Berre (1998, p. 44), de la façon suivante: "l'inscription des divers phénomènes culturels dans le canevas d'ensemble qui leur donne un cadre et rend possible leur compréhension, permet de construire une véritable systématique de la civilisation étudiée."

L'importance de placer les phénomènes culturels dans leur contexte pour comprendre une culture, est concomitante de l'importance indiscutable du contexte des interactions sociales, lieu où la langue, la culture et les représentations interagissent, communiquent et produisent du sens.

Selon Cuche (2010, p. 50), la pluralité des contextes d'interaction explique le caractère pluriel et instable de toute culture, et aussi les comportements apparemment contradictoires d'un même individu. Ainsi comme la langue est le produit de l'interaction entre ceux qui la parlent, la culture, selon Cuche (2010, p. 49), n'existe que par le jeu interactif des individus. Selon Abdallah-Pretceille e Porcher (2005, p. 48)

La culture est un outil dont nous nous servons pour être et vivre dans le monde. C'est un moyen de communication qui s'actualise dans le comportement, dans les actions, dans le discours, dans l'interaction sociale. C'est un processus dynamique, susceptible de plusieurs lectures interprétatives et des expressions plurielles et, surtout, la culture est l'expression d'un point de vue susceptible d'être confirmé ou réfuté par d'autres versions. 
Ainsi, langue et culture s'actualisent, construisent des significations et présentent leur dynamique dans la diversité des contextes, des relations et des interactions sociales. En ce sens, la langue nous donne accès à la culture dans sa dimension sociologique, puisque nous en avons besoin pour interagir, et c'est dans les interactions qu'entrent en jeu les représentations dont nous nous servons pour agir et réagir avec nos interlocuteurs.

\section{Références}

ABDALLAH-PRETCEILLE, M.; PORCHER, L. Education et communication interculturelle. Paris: Presses Universitaires de France, 2005.

ALVAREZ, G. La notion de Français Instrumental. Langues et Linguistique, Québec, v. 2, p. 1-9, 1976.

CANDIDO, A. O francês instrumental. Paris: Hemus, 1977.

CONSEIL DE L'EUROPE. Cadre européen commun de référence pour l'apprentissage et l'enseignement des langues. Paris: Didier, 2001.

CUCHE, D. La notion de culture dans les sciences sociales. Paris: La Découverte, 2010.

DE CARLO, M.; LANCIEN, T. L 'interculturel. Paris: Clé International, 1998.

GERMAIN, C. Evolution dans l'enseignement de langues. 5000 ans d'histoire. Paris: Clé International, 1993.

GERMAIN, C. La didactique des langues: les relations entre les plans psychologique, linguistique et pédagogique. Linguarum Arena, Porto, v. 1, n. 1, p. 9-24, 2010.

LE BERRE, M.-B. Concepts et outils en maîtrise FLE pour une éducation à la perception interculturelle au CUEF/Université Sthendal. L'apport des Centres de Français Langue Etrangère à la Didactique des Langues, LIDIL, Grenoble, v. 16, p. 38-59, 1998.

MONTREDON, J. et al. C'est le printemps. Paris: Clé International, 1976.

PORCHER, L. L'enseignement de la civilisation. Revue Française de Pédagogie, Persée, v. 108, n. 1, p. 5-12, 1994. 
PORCHER, L. L'Europe: objet d'enseignement? In: JOURNEES D'ETUDE DE L'AFDECE, CIEP, 2004, Sèvres. Actes... Paris: [s.n.], 2004. p. 96-121

PUREN, C. De la méthodologie à la didactologie: hommage à Robert Galisson. Paris: Didier Erudition, 2001.

TROTOT. [Apresentação]. In: LÉRY, J. Histoire d'un voyage fait en la terre de Brésil 1578. Paris: Éditions LGF, 1998. (Collection LDP, 707).

ZARATE, G. Enseigner une culture étrangère. Paris: Hachette, 1986. 